\title{
https://doi.org/10.46813/2020-130-036 \\ TO THE DISTRIBUTION OF THE DISPERSION EQUATION ROOTS FOR THE PARAMETRIC CHERENKOV RADIATION
}

\author{
V.I. Tkachenko ${ }^{1,2}$, I.V. Tkachenko ${ }^{1}$, A.P. Tolstoluzhsky ${ }^{1}$, S.N. Khizhnyak ${ }^{1}$ \\ ${ }^{1}$ National Science Center "Kharkov Institute of Physics and Technology”, Kharkiv, Ukraine; \\ ${ }^{2}$ V.N. Karazin Kharkiv National University, Kharkiv, Ukraine
}

\section{E-mail: tkachenko@kipt.kharkov.ua}

\begin{abstract}
Distribution of the roots of dispersion equation of the parametric Cherenkov radiation (PCR) excited by uniformly moving charged particle in an ideally conducting metal waveguide filled with a spatially periodic layered dielectric is studied. The study both in long wavelengths range and in the range of wavelengths comparable and smaller than the period of non-uniformity is presented. It is shown that in the case of long wavelengths the dependence of the frequency of the excited PCR on the particle velocity is monotonic. For the case of wavelengths comparable and shorter than the non-uniformity period the spectrum of PCR is more complicated. Its characteristic features are determined.
\end{abstract}

PACS: 41.60.-m

\section{INTRODUCTION}

The effect of electromagnetic wave radiation during the motion of a charged particle at a constant velocity in the metal waveguide filled with a spatially periodic layered dielectric was first studied in the paper [1]. The authors called this radiation as parametric Cherenkov radiation (PCR). The results of the study of this radiation are presented in voluminous literature (see, for example, [2-11] and the literature cited therein). Studying different aspects of the PCR, various authors gave it different names - resonant transition radiation, $\mathrm{X}$-ray transition radiation, parametric $\mathrm{X}$-ray radiation and others. Since the general condition for radiation occurrence is the periodic variation of parameters, we will follow the name PCR.

Since there is no analytical solution of the dispersion equation for PCR in an interesting for practical applications wavelengths (comparable or shorter than the non-uniformity period), the analytical expressions to calculate the generated fields and radiation power of the assumed microwave generator can not be evaluated.

In this work the analytical solution of the dispersion equation for the PCR in a waveguide loaded with layered dielectric in the wavelength range of comparable and smaller than the non-uniformity period along with the case of long wavelength range is obtained and compared with the results of numerical calculations presented in [11].

\section{ANALYTICAL STUDY OF SOLUTIONS OF THE DISPERSION EQUATION OF PCR}

Let us consider layered medium involved alternating layers of two homogeneous and isotropic dielectrics. In each longitudinal period $L=a+b$ the first layer $0 \leq z \leq a$ has dielectric and magnetic permeabilities $\varepsilon_{1}, \mu_{1}$, the second layer $a \leq z \leq b$ has permeabilities $\varepsilon_{2}, \mu_{2}$. Dielectrics are in a cylindrical perfectly conductive metal waveguide.

The frequencies of the electromagnetic radiation of a charged particle, moving with velocity $v$ in such medium, are determined by the roots of the dispersion equation [1]:

$$
\cos (k L / \beta)-g(\psi)=0,
$$

where

$g(\psi)=\cos \left(p_{1} a\right) \cos \left(p_{2} b\right)-\frac{1}{2}\left(\frac{p_{1} \varepsilon_{2}}{p_{2} \varepsilon_{1}}+\frac{p_{2} \varepsilon_{1}}{p_{1} \varepsilon_{2}}\right) \sin \left(p_{1} a\right) \sin \left(p_{2} b\right)$ and the following designations are introduced $p_{1}=\sqrt{\varepsilon_{1} \mu_{1} k^{2}-k_{\perp}^{2}}$ is longitudinal wave number in the first layer, $p_{2}=\sqrt{\varepsilon_{2} \mu_{2} k^{2}-k_{\perp}^{2}}$ is longitudinal wave number in the second layer, $k=\omega / c, k_{\perp}=\alpha_{n} / R$ is transverse wave number, $R$ is waveguide radius, $\alpha_{n}$ is $n$-root of the zero-order Bessel function, $\beta=v / c$ is dimensionless velocity of the charged particle.

Since the equation (1) is a dispersion equation of the radiation arising in the layered dielectric (medium with periodically varying parameters), the frequencies determined by the roots of this equation correspond to the waves propagating in such layered medium.

It can be seen that the solutions of the dispersion equation should satisfy the condition: $-1 \leq g(\psi) \leq 1$.

It is easy to show that at $\varepsilon_{1} \rightarrow \varepsilon_{2}, \mu_{1} \rightarrow \mu_{2}$ the value $\frac{1}{2}\left(\frac{p_{1} \varepsilon_{2}}{p_{2} \varepsilon_{1}}+\frac{p_{2} \varepsilon_{1}}{p_{1} \varepsilon_{2}}\right) \rightarrow 1$ and the equation (1) takes the form

$$
\cos (\omega L / v)-\cos (p L)=0 .
$$

The solution of this equation gives the condition of the Cherenkov radiation of the charged particle at a uniform motion through the dielectric waveguide filled with homogeneous dielectric

$$
\omega=k_{z} v, \text { where } k_{z}=p=\sqrt{\varepsilon \mu k^{2}-k_{\perp}^{2}} .
$$

Since we consider waves propagating in the waveguide with dielectric disks, the function $g(\psi)$ is a real function of frequency and can be represented as the sum of two cosines

$$
g(\psi)=\cos \left(p_{1} a+p_{2} b\right) \cdot(1+\delta)-\cos \left(p_{2} b-p_{1} a\right) \cdot \delta,
$$


where $\delta=\frac{\left(p_{1} \varepsilon_{2}-p_{2} \varepsilon_{1}\right)^{2}}{4 p_{1} \varepsilon_{2} p_{2} \varepsilon_{1}}$.

In the general case, the quantities $p_{1}$ and $p_{2}$ are incommensurable quantities in the space of wave numbers $\mathrm{k}$. The function $\delta$ in the expression (4) depends in a complicated way on the parameters of the dielectric waveguide and frequency. Therefore, as a result, the sum of terms in the right-hand side of (4) cannot have a common period and to study analytically the solutions of the dispersion equation (1) is not possible.

But only in some special cases it may be done. For example, in the case of sufficiently thin disks, when the conditions $p_{1} a<<1, p_{2} b<<1$ as well as $k L / \beta<<1$ (long-wave approximation) are satisfied, analytical solutions of equation (1) can be found.

In this case, taking into account the quadratic terms $\left(p_{1} a\right)^{2},\left(p_{2} b\right)^{2},(k L / \beta)^{2}$, the function $g(\psi)$ is identical to the expression

$$
g(\psi)=1-\frac{1}{2}\left(p_{1}^{2} a^{2}+p_{2}^{2} b^{2}+\frac{a \cdot b \cdot\left(\varepsilon_{1}^{2} \cdot p_{2}{ }^{2}+\varepsilon_{2}^{2} \cdot p_{1}^{2}\right)}{\varepsilon_{1} \cdot \varepsilon_{2}}\right)
$$

and the dispersion equation (1) has a form [1]:

$$
\frac{k^{2}}{\beta^{2}}=\frac{\varepsilon_{\perp}}{\varepsilon_{p}}\left(\varepsilon_{p} k^{2}-k_{\perp}^{2}\right),
$$

where $\varepsilon_{\perp}=\frac{(a+b) \varepsilon_{1} \mu_{1} \varepsilon_{2} \mu_{2}}{\left(a \varepsilon_{2} \mu_{2}+b \varepsilon_{1} \mu_{1}\right)}, \varepsilon_{p}=\frac{\left(a \varepsilon_{1} \mu_{1}+b \varepsilon_{2} \mu_{2}\right)}{(a+b)}$.

The solution of equation (5) explicitly establishes the correspondence between the wave number $k$ and particle velocity $\beta$ at fixed values of the parameters of the medium and the diameter of the waveguide:

$$
\beta= \pm \frac{k \sqrt{\varepsilon_{p}}}{\sqrt{\varepsilon_{\perp}} \sqrt{k^{2} \varepsilon_{p}-k_{\perp}^{2}}}, k= \pm \frac{\beta k_{\perp}}{\sqrt{\beta^{2} \varepsilon_{\perp}-1}} \sqrt{\frac{\varepsilon_{\perp}}{\varepsilon_{p}}} .
$$

As it can be seen from (6), there is a monotonic dependence of the wave number $k$ of the excited PCR on the particle velocity $\beta$ in the long-wave approximation. In this case, PCR occurs when the particle velocity is exceeded the threshold velocity value $\beta>\beta_{\min }=1 / \sqrt{\varepsilon_{\perp}}$. Depending on the degree of filling by the layers with a dielectric and the width of the layers, the value $\varepsilon_{\perp}$ lies within the range $\varepsilon_{1} \leq \varepsilon_{\perp} \leq \varepsilon_{2}$.

Fig. 1 shows the dependences of $k(\beta) \cdot L$ on $\beta$ for two values of the spatial period of the disks $L-L=2.1 \cdot 10^{-1}$ and $2.1 \cdot 10^{-2} \mathrm{~cm}$.

It should be noted, that as the layer width decreases the usability condition for dispersion equation (5) expands to the high-frequency area.

On the other hand, for sufficiently large values of $\varepsilon_{i} \mu_{i} \cdot k^{2}>>k_{\perp}^{2} \quad(i=1,2)$, up to quadratic terms $\frac{k_{\perp}{ }^{2}}{\varepsilon_{i} \mu_{i} k^{2}}$, the dispersion equation (1) can be represented as:

$$
\begin{gathered}
\cos (k L / \beta)=\cos \left[\left(\sqrt{\varepsilon_{1} \mu_{1}} \cdot a+\sqrt{\varepsilon_{2} \mu_{2}} \cdot b\right) k\right] \cdot(1+\delta)- \\
-\cos \left[\left(\sqrt{\varepsilon_{2} \mu_{2}} \cdot b-\sqrt{\varepsilon_{1} \mu_{1}} \cdot a\right) k\right] \cdot \delta,
\end{gathered}
$$

where $\delta=\frac{1}{4} \frac{\left(\sqrt{\varepsilon_{1} \mu_{1}} \varepsilon_{2}-\sqrt{\varepsilon_{2} \mu_{2}} \varepsilon_{1}\right)^{2}}{\sqrt{\varepsilon_{1} \mu_{1}} \varepsilon_{2} \sqrt{\varepsilon_{2} \mu_{2}} \varepsilon_{1}}$.

In this approximation the function $g(\psi)$ is a periodic one with two periods in the wave number $k$ :

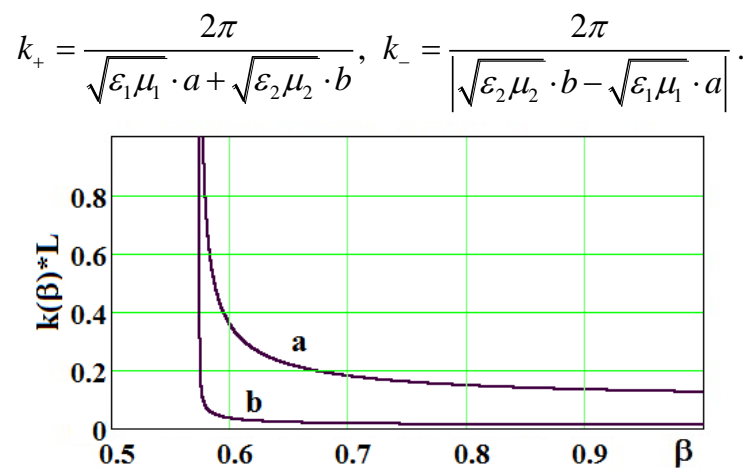

Fig. 1. The dependence of $k(\beta) \cdot L$ on particle velocity $\beta$. For $\mu_{1}=\mu_{2}=1, \varepsilon_{1}=1.96, \varepsilon_{2}=4, k_{\perp}=\alpha_{1} / R$, $R=3 \mathrm{~cm} ; \boldsymbol{a}-L=2.1 \cdot 10^{-1} \mathrm{~cm}$ at $a=1 \cdot 10^{-1} \mathrm{~cm}$, $b=1.1 \cdot 10^{-1} \mathrm{~cm} ; \boldsymbol{b}-L=2.1 \cdot 10^{-2} \mathrm{~cm}$ at $a=1 \cdot 10^{-2} \mathrm{~cm}$, $b=1.1 \cdot 10^{-2} \mathrm{~cm}$

In this case, the term with the wave number $k_{+}$ corresponds to the wavelength $\lambda_{+}=\sqrt{\varepsilon_{1} \mu_{1}} a+\sqrt{\varepsilon_{2} \mu_{2}} b$, and the term with the wave number $k_{-}$corresponds to the wavelength $\lambda_{-}=\left|\sqrt{\varepsilon_{2} \mu_{2}} \cdot b-\sqrt{\varepsilon_{1} \mu_{1}} \cdot a\right|$.

Thus, in the case under consideration the dispersion equation of the PCR (1) is a periodic function on $k$ with three spatial periods $\lambda_{+}, \lambda_{-}, \lambda_{L}=L / \beta$.

To explain the phenomenon of superposition of three waves, we will find a solution of equation (7).

From the equation (7) it follows that for sufficiently large values $k$ it is satisfied at some fixed values $k_{i}$, where $k_{i}$ are the roots of equation (7). Since the quantity $\delta$ does not depend on $k$, then the possible roots of equation (7) are those for which the following identities are valid:

$$
\begin{aligned}
\cos \left(k_{i} L / \beta\right) & =\cos \left[\left(\sqrt{\varepsilon_{1} \mu_{1}} \cdot a+\sqrt{\varepsilon_{2} \mu_{2}} \cdot b\right) k_{i}\right]= \\
& =\cos \left[\left(\sqrt{\varepsilon_{2} \mu_{2}} \cdot b-\sqrt{\varepsilon_{1} \mu_{1}} \cdot a\right) k_{i}\right]
\end{aligned}
$$

Thus, the solutions of equation (7) are satisfied at the following relations between the arguments for all equalities (8):

$$
\begin{aligned}
k_{i} L / \beta+2 \pi j & =\left(\sqrt{\varepsilon_{1} \mu_{1}} \cdot a+\sqrt{\varepsilon_{2} \mu_{2}} \cdot b\right) k_{i}+2 \pi m= \\
& =\left(\sqrt{\varepsilon_{2} \mu_{2}} \cdot b-\sqrt{\varepsilon_{1} \mu_{1}} \cdot a\right) k_{i}+2 \pi n
\end{aligned}
$$

where $j, m, n$ are arbitrary integers.

Therefore, it is not difficult to find a relation determining the values of a certain sequence of solutions of the dispersion equation satisfying the conditions (8):

$$
k_{N}=C\left(a, b, \beta, \varepsilon_{1}, \mu_{1}\right) \cdot N,
$$

where $C\left(a, b, \beta, \varepsilon_{1}, \mu_{1}\right)=\frac{\pi}{L / \beta-\sqrt{\varepsilon_{2} \mu_{2}} \cdot b}, N=(n-j)$. 
To verify the validity of the obtained solutions, it is necessary to substitute the values of the roots from (10) into the original dispersion equation (1) and find by numerical analysis the values of $N$ at which $k_{N}$ is the solutions of the original dispersion equation.

A numerical study of solutions of the PCR dispersion equation and comparison of the analytical solutions (9) of the dispersion equation (1) with the results of its numerical analysis were carried out below.

\section{NUMERICAL STUDY OF SOLUTIONS OF THE DISPERSION EQUATION OF PCR}

Graphs of the dependence of the function $D(k)=\cos (k L / \beta)-g(\psi)=0$ on $k$ and its spectrum are presented by curves of Fig. 2, 7 for non-magnetic media $\quad \mu_{1}=\mu_{2}=1, \quad \varepsilon_{1}=1.96, \quad \varepsilon_{2}=4, \quad a=1 \cdot 10^{-1} \mathrm{~cm}$, $b=1.10 \cdot 10^{-1} \mathrm{~cm}, R=3 \mathrm{~cm}$ for two values of the particle velocity $\beta=0.7$ (see Fig. 2), $\beta=0.58333$ (Fig. 7). They show, that the dependence $D(k)$ is determined, as one would expect, by the interference (beating) of three cosine waves (see (1), (7)) at a wavelength $\lambda_{L}=L / \beta$ equal to the characteristic length of the $\cos (k L / \beta)$ term variation and the wavelengths $\lambda_{+}, \lambda$, corresponding to the characteristic lengths of $g(\psi)$ variation.
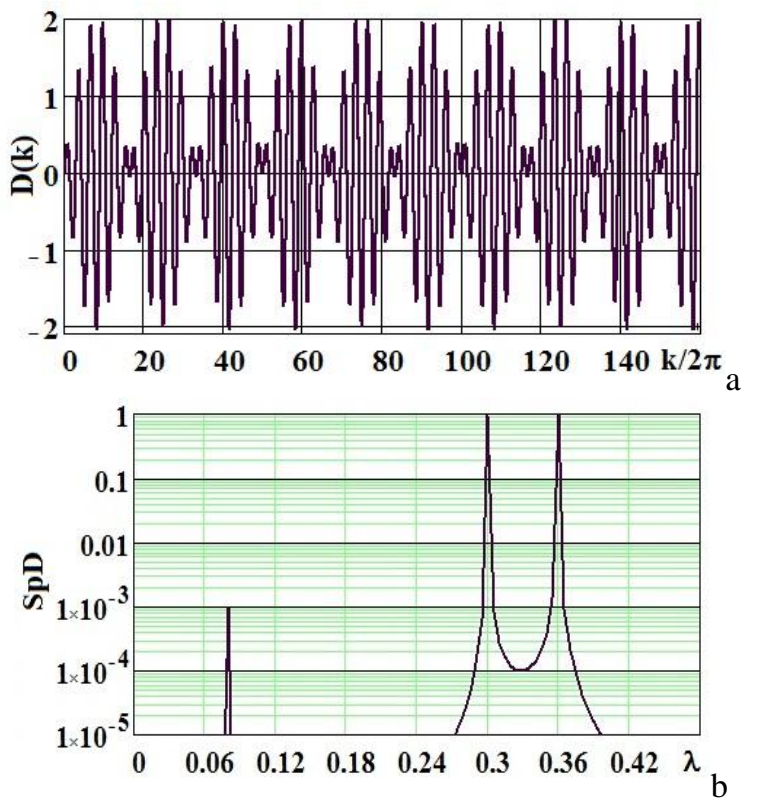

Fig. 2. The dependence $D(k)=\cos (k L / \beta)-g(\psi)$ on $k(a)$ and its spectrum $S p D$ on $\lambda=2 \pi / k(b)$ for

$$
\beta=0.7, k_{\perp}=\alpha_{1} / R=2.404 / 3=0.80133 \mathrm{~cm}^{-1}
$$

As can be seen from these diagrams, the characteristic values of wavelengths $\lambda_{L}, \lambda_{+}, \lambda_{-}$for the excited PCR found analytically coincide with sufficient accuracy with their values obtained by numerical analysis $-\lambda_{L},=0.3 \mathrm{~cm}, \lambda_{+}=0.36 \mathrm{~cm}, \lambda_{-}=0.08 \mathrm{~cm}$. Further, we assume that all spatial variables are measured in $\mathrm{cm}$ and the wave numbers $k-\mathrm{cm}^{-1}$.

To determine the spectral characteristics of the radiation resulting from the interference of three waves $\lambda_{L}, \lambda_{+}, \lambda_{-}$, the distribution of the roots of the dispersion equation for the particle velocity $\beta=0.7$ was numerically studied.

Fig. 3 shows the dependence of the sequence of wave number values $k_{i}$ for the roots of the dispersion equation $D\left(k_{i}\right)=0$ as a function of the root number $i$.

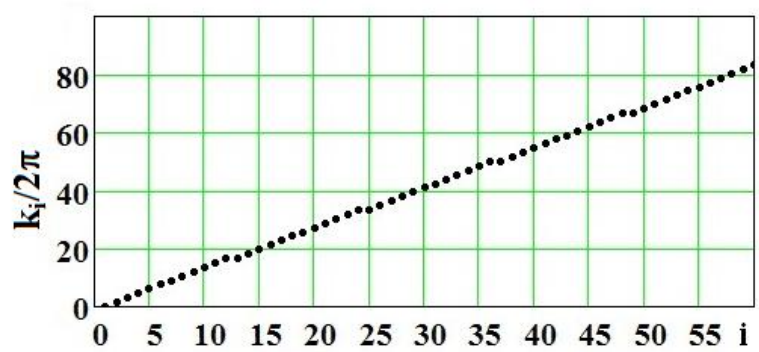

Fig. 3. The dependence of the sequence of wave number values $k_{i} / 2 \pi$ for the roots of the dispersion equation $D\left(k_{i}\right)=0$ of layered dielectric on the root number $i$ for $\beta=0.7$

From Fig. 3 it is shown, that there is a violation of the monotonicity of following of the wave numbers values $k_{i}$, which consists in a periodic transition from one straight line to another located below.

To clarify the nature of this nonmonotonicity the dependence of the difference of neighboring roots of the dispersion equation $D(k)=\cos (k L / \beta)-g(\psi)$ on the values of these roots is presented in Fig. 4 .

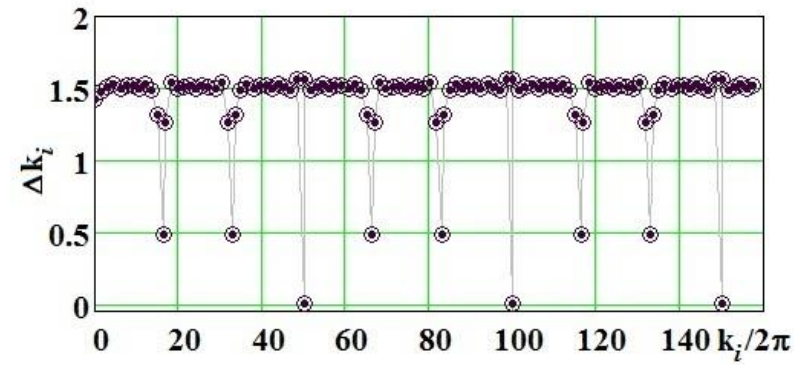

Fig. 4. The dependences of the difference

$\Delta k_{i}=\left(k_{i}-k_{i-1}\right) / 2 \pi$ of neighboring roots of the dispersion equation $D\left(k_{i}\right)=0$ of a layered dielectric on the values of these roots $k_{i} / 2 \pi$ for $\beta=0.7$

As it can be seen from this figure, a strictly periodic alternation of the difference of the roots of the equation (1) is observed. Besides, as the numerical analysis shows, there is a peculiarity in the values of the roots of this equation - almost coincidence of some two neighboring roots at a level of order $\Delta k_{i} \sim 10^{-4}$ with values $k$ near $k_{36} / 2 \pi=50, k_{72} / 2 \pi=100$ and $k_{108} / 2 \pi=150$. With the same periodicity $k / 2 \pi=50$ the remaining roots of the dispersion equation alternate. At the same time, as frequency increases, character of root following is preserved.

Fig. 5 shows the dependence $D\left(k_{N}\right)$ as a function of the number $N$ for the particle velocity $\beta=0.7$ obtained from the formula (2).

As can be seen from this figure, the values $k_{N}$ are the roots of the dispersion equation (1) for the values $N=8,16,24, \ldots$ At that, $L / \beta-\sqrt{\varepsilon_{2} \mu_{2}} \cdot b=0.08$, 
$C\left(a, b, \beta, \varepsilon_{l}, \mu_{l}\right)=12.5 \pi$. In this case analytical roots periodicity $k_{\text {analit }}=8 C\left(a, b, \beta, \varepsilon_{l}, \mu_{1}\right) / 2 \pi=50$, root periodicity obtained numerically: $k_{\text {num }} \approx 49.99995$

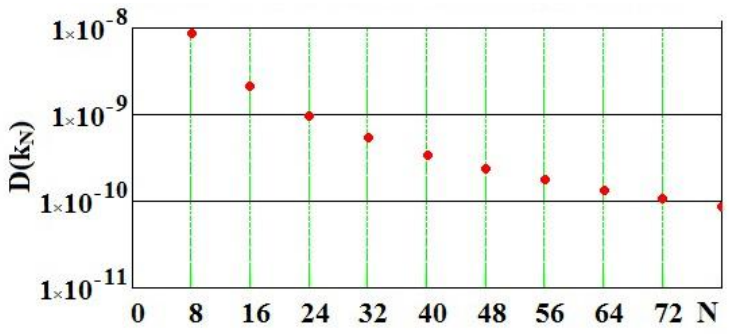

Fig. 5. The dependence $D\left(k_{N}\right)$ as a function of the number $N$ for $\beta=0.7$

Thus, the values of the roots of the dispersion equation (1) obtained by the formula (10) $k_{N}=C\left(a, b, \beta, \varepsilon_{l}, \mu_{l}\right) \cdot N$ correspond with a sufficient degree of accuracy to the values of the roots obtained by numerically solving this equation: $k_{N=8}=k_{36}$, $k_{N=16}=k_{72} ., k_{N=24}=k_{108}$. Note, that with increasing the number $N$ the accuracy of calculating the expression (10) increases and the relation between the roots follows exactly the relations for periods $\lambda_{L}, \lambda_{+}, \lambda_{-}$:

$$
\frac{\lambda_{+}}{\lambda_{-}}=\frac{p_{1} a+p_{2} b}{p_{2} b-p_{1} a}, \quad \frac{k L / \beta}{p_{2} b-p_{1} a}=\frac{\lambda_{L}}{\lambda_{-}}, \frac{\lambda_{+}}{\lambda_{L}}=\frac{p_{1} a+p_{2} b}{k L / \beta} .
$$

This is confirmed by the data given in Fig. 6 .

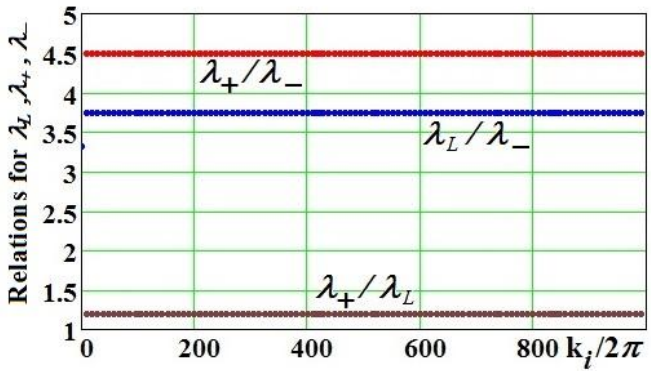

Fig. 6. The dependence of relations $\lambda_{+} / \lambda_{-}=4.5$, $\lambda_{L} / \lambda_{-}=3.75$ and $\lambda_{+} / \lambda_{L}=1.2$ upon roots $k_{i} / 2 \pi$ for $\beta=0.7$

From the relations for periods $\lambda_{L}, \lambda_{+}, \lambda_{-}$it follows that, it is possible, for example, to superpose the periods $\lambda_{L}$ and $\lambda_{+}$by varying the particle velocities. So, at $k L \gg>1$ and the particle velocity $\beta=\frac{k L}{p_{1} a+p_{2} b}=0.5833333$, these periods are equal $\lambda_{L}=\lambda_{+}$.

It follows from Fig. 7 that wavelengths $\lambda_{L}, \lambda_{+}$ coincide $-\lambda_{L}=\lambda_{+}=0.36, \lambda_{-}=0.08$.

As it follows from Fig. 8, there is a strictly periodic alternation of the difference of roots of the equation (1). It should be added that the numerical analysis shows almost of some two neighboring roots with an accuracy of the order $\Delta k_{i} \sim 10^{-3}$.

The dependence $D\left(k_{N}\right)$ as a function of the number $N$ for a particle velocity $\beta=0.58333$ obtained by the formula (10) is presented in Fig. 9.

As it can be seen from this figure the values $k_{N}$ are the roots of the dispersion equation (1) for the values $N=7, \quad 14, \quad 21, \ldots \quad$ At that, $L / \beta-\sqrt{\varepsilon_{2} \mu_{2}} \cdot b=0.14$, $C\left(a, b, \beta, \varepsilon_{l}, \mu_{l}\right)=50 \pi / 7$. In this case analytical roots periodicity $\kappa_{\text {analit }}=14 C\left(a, b, \beta, \varepsilon_{1}, \mu_{1}\right) / 2 \pi=50$ of $\operatorname{root}$ is equal to periodicity obtained numerically: $k_{\text {num }} \approx$ 49.99995.
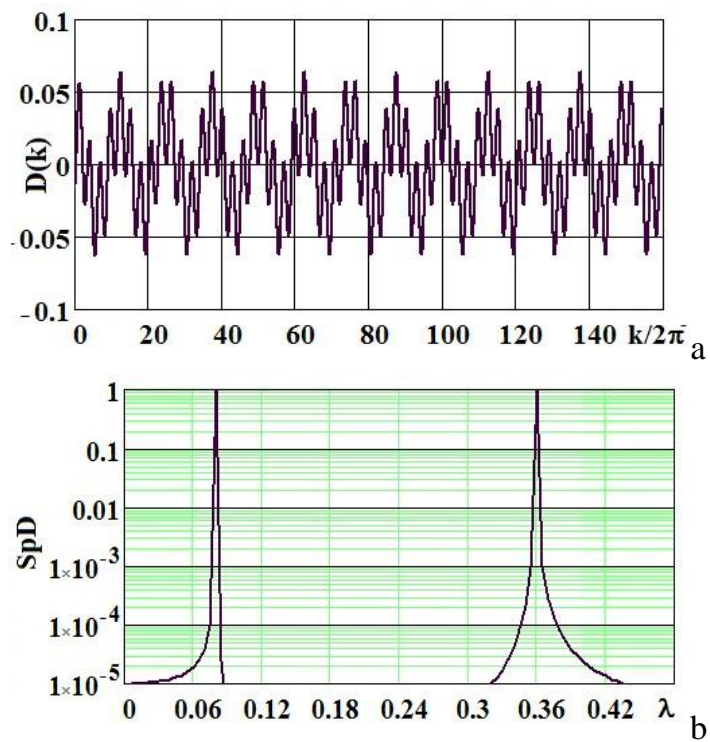

Fig. 7. The dependence $D(k)=\cos (k L / \beta)-\cos (\psi)$ on $k=\omega / c(a)$ and its spectrum $\operatorname{SpD}(b)$ on $\lambda=2 \pi / k$ for $\beta=0.583333, k_{\perp}=\alpha_{1} / R$

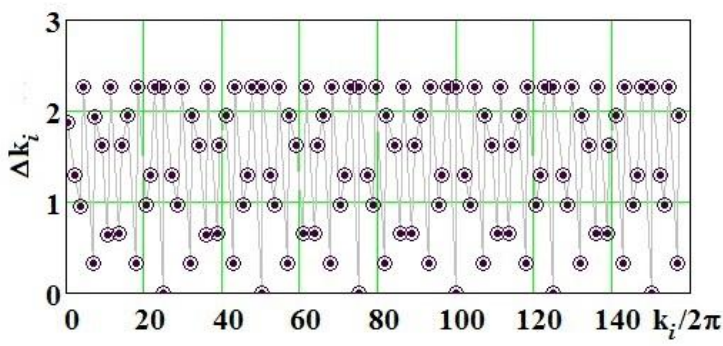

Fig. 8. The dependence of difference

$\Delta k_{i}=\left(k_{i}-k_{i-1}\right) / 2 \pi$ of neighboring roots of the dispersion equation $D\left(k_{i}\right)=0$ on the values of these roots

$$
k_{i} / 2 \pi \text { for } \beta=0.58333
$$

Thus, the values of roots of the dispersion equation (1) obtained by the formula (10) $k_{N}=C\left(a, b, \beta, \varepsilon_{l}, \mu_{l}\right) \cdot N$ correspond with sufficient degree of accuracy to the values of the roots obtained by numerically solving of the equation (1): $k_{N=7}=k_{36}, k_{N=14}=k_{72}, k_{N=21}=k_{108}$. At that, the relation between the roots exactly follows the relations between the periods of the waves $\lambda_{L}, \lambda_{+}, \lambda_{-}$:

$\lambda_{+} / \lambda_{-}=4.5, \lambda_{+} / \lambda_{\mathrm{L}}=1$.

Calculations show that with increasing frequency, the character of following of the roots is preserved.

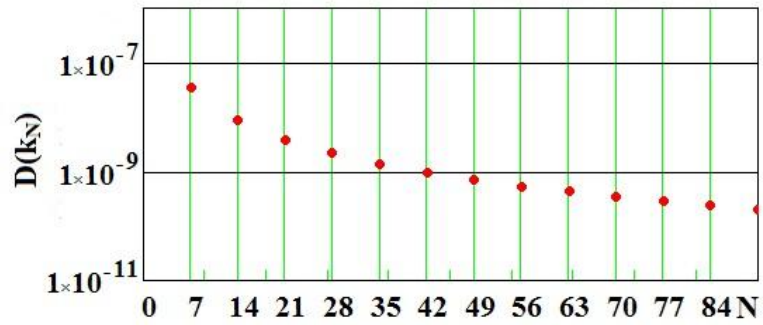

Fig. 9. The dependence $D\left(k_{N}\right)$ as a function of number $N$ for $\beta=v / c=0.58333$ 


\section{CONCLUSIONS}

Thus, as a result of the analytical and numerical study of the dispersion equation of the PCR of a charged particle moving along the axis of an ideally conducting metal waveguide filled with a spatially periodic layered dielectric, the following conclusions can be drawn.

1. In the range of wavelengths greater than nonuniformity period $(k L / \beta<<1)$ the frequency of PCR depends monotonically on the particle velocity. Under condition $k L / \beta>>1$, at fixed particle velocity $\beta$, spectrum of PCR is discrete. The dependence of values of the root sequence of the dispersion equation on the root number is non-monotonic. In this case, the difference of the roots of dispersion equation alternate with a certain periods, the values of these periods are determined numerically.

2 . The analytical solution of the dispersion equation of the PCR at $\varepsilon_{i} \mu_{i} \cdot k^{2}>>k_{\perp}^{2}$ was obtained and the periodic discrete nature of the spectrum caused by the presence of three groups of wave numbers in the dispersion equation (8) was revealed. The values of the roots obtained analytically are in good coincidence with the values of the roots determined numerically.

3. A peculiarity in the values of the neighboring roots of the dispersion equation - the coincidence of some two neighboring roots at the level of the order $\Delta k_{i} \sim 10^{-3}$ was found.

\section{ACKNOWLEDGMENTS}

The authors express their gratitude to Professor I.N. Onishchenko for fruitful discussions and useful comments.

\section{REFERENCES}

1. Ya.B. Fainberg, N.A. Khizhnyak. Energy Loss of a Charged Particle Passing Through a Laminar Dielectric // Soviet Physics JETP. 1957, v. 5, № 4, p.720-729.

2. V.L. Ginzburg, V.N. Tsytovich. Several problems of the theory of transition radiation and transition scattering // Physics Reports. 1979, v. 49, № 1, p.1-89.
3. V.L. Ginzburg. Radiation by uniformly moving sources (Vavilov-Cherenkov effect, transition radiation, and other phenomena) // Sov. Phys. Usp. 1996, v. 39, № 10, p. 973-982.

4. V.L. Ginzburg, V.N. Tsytovich. Transition Radiation and Transition Scattering. Moscow: "Nauka", 1983, p. 360 (in Russian). English translation: A Hilger, New York: "Bristol", 1990.

5. M.L. Ter-Mikaelyan. The influence of the medium on electromagnetic processes at high energies. AN Arm. SSR. 1969, p.457.

6. G.M. Garibyan, Yan Shi. X-ray transition radiation. Yerevan: Issue. AN ArmSSR, 1983, p. 420 (In Russian).

7. F.G. Bass, V.M. Yakovenko. Theory of radiation from a charge passing through an electrically inhomogeneous medium // Sov. Phys. Usp. 1965, v. 8, № 3, p.420-444.

8. Ya.B. Fainberg. N.A. Khizhnyak. The phenomenon of electromagnetic wave radiation by a charged particle uniformly and rectilinearly moving in a spatially periodic medium (parametric Cherenkov effect) // Plasma electronics. Kyiv: «Naukova Dumka», 1989, $300 \mathrm{p}$.

9. V.A. Buts, N.A. Khizhnyak. Study of parametric Cherenkov radiation // Plasma electronics. Kyiv: «Naukova Dumka», 1989, 300 p.

10. V.A. Buts. Parametric Cherenkov radiation (idei development) // Problems of Atomic Science and Technology. Series «Plasma Electronics and New Methods of Acceleration» (4). 2004, № 4, p. 70-75.

11. V.I. Tkachenko, I.V. Tkachenko, A.P. Tolstoluzhsky, S.N. Khizhnyak. Radiation of a charged particle in the ideally conducting metal waveguide filled with a spatially periodic layered dielectric // Problems of Atomic Science and Technology. Series «Plasma Electronics and New Methods of Acceleration». 2018, № 4, p. 13-16

Article received 18.09.2020

\section{О РАСПРЕДЕЛЕНИИ КОРНЕЙ ДИСПЕРСИОННОГО УРАВНЕНИЯ ПАРАМЕТРИЧЕСКОГО ЧЕРЕНКОВСКОГО ИЗЛУЧЕНИЯ}

\section{В. И. Ткаченко, И.В. Ткаченко, А.П. Толстолужский, С.Н. Хижняк}

Исследуется распределение корней дисперсионного уравнения параметрического черенковского излучения (ПЧИ), возбуждаемого равномерно движущейся заряженной частицей в идеально проводящем металлическом волноводе, заполненном пространственно-периодическим слоистым диэлектриком как в области длинных волн, так и в диапазоне длин волн, сопоставимых и меньших, чем период неоднородности. Показано, что в случае длинных волн зависимость частоты возбуждаемого ПЧИ от скорости частицы является монотонной. В случае длин волн, сравнимых и более коротких, чем период неоднородности, спектр ПЧИ более сложный. Определены его характерные особенности.

\section{ПРО РОЗПОДІЛ КОРЕНІВ ДИСПЕРСІЙНОГО РІВНЯННЯ ПАРАМЕТРИЧНОГО ЧЕРЕНКОВСЬКОГО ВИПРОМІНЮВАННЯ}

\section{В. І. Ткаченко, І.В. Ткаченко, О.П. Толстолужський, С.М. Хижнняк}

Досліджується розподіл коренів дисперсійного рівняння параметричного черенковського випромінювання (ПЧВ), збуджуваного зарядженою частинкою, що рівномірно рухається в ідеально провідному металевому хвилеводі, заповненому просторово-періодичним шаруватим діелектриком як в області довгих хвиль, так і в діапазоні довжин хвиль, порівнянних і менших, ніж період неоднорідності. Показано, що у випадку довгих хвиль залежність частоти збуджуваного ПЧВ від швидкості частинки $є$ монотонною. У випадку довжин хвиль, порівнянних і більш коротких, ніж період неоднорідності, спектр ПЧВ більш складний. Визначені його характерні особливості. 